\title{
DIMENSION DEPENDENCE OF FACTORIZATION PROBLEMS: HARDY SPACES AND $S L_{n}^{\infty}$
}

\author{
RICHARD LECHNER
}

\begin{abstract}
Given $1 \leq p<\infty$, let $W_{n}$ denote the finite-dimensional dyadic Hardy space $H_{n}^{p}$, its dual or $S L_{n}^{\infty}$. We prove the following quantitative result: The identity operator on $W_{n}$ factors through any operator $T: W_{N} \rightarrow W_{N}$ which has large diagonal with respect to the Haar system, where $N$ depends linearly on $n$.
\end{abstract}

\section{INTRODUCTION}

Local theory of Banach spaces is concerned with the quantitative study of finite dimensional Banach spaces and their relation to infinite dimensional spaces and operators. To illustrate, we give the following example.

Suppose that for each $n \in \mathbb{N}$, the $n$-dimensional Banach space $X_{n}$ has a normalized 1-unconditional basis $e_{j}, 1 \leq j \leq n$, and let $e_{j}^{*} \in X_{n}^{*}, 1 \leq j \leq n$ denote the associated coordinate functionals.

Question 1.1. Given $n \in \mathbb{N}$ and $\delta, \Gamma, \eta>0$, what is the smallest integer $N=$ $N(n, \delta, \Gamma, \eta)$, such that for any operator $T: X_{N} \rightarrow X_{N}$ satisfying

$$
\|T\| \leq \Gamma \quad \text { and } \quad\left|\left\langle e_{j}^{*}, T e_{j}\right\rangle\right| \geq \delta, \quad 1 \leq j \leq N,
$$

there are there operators $E: X_{n} \rightarrow X_{N}$ and $F: X_{N} \rightarrow X_{n}$ such that the diagram

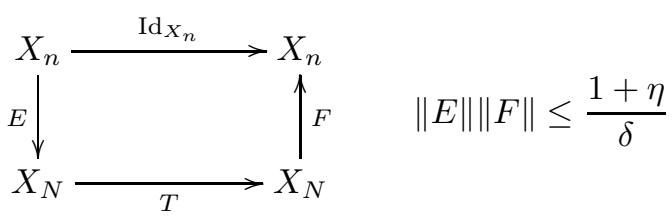

is commutative?

Note that the diagonal operator $D: X_{n} \rightarrow X_{n}$ given by $D=\delta \operatorname{Id}_{X_{n}}$, where $\operatorname{Id}_{X_{n}}$ denotes the identity operator on $X_{n}$, shows that for every choice for $E$ and $F$ we have $\|E\|\|F\| \geq \frac{1}{\delta}$.

Naturally, we are interested in estimates for $N=N(n, \delta, \Gamma, \eta)$, especially in the relation between $N$ and $n$. For many Banach spaces, we have quantitative estimates for $N$ (see e.g. 2, 3, 12, 1, 15, 14, 11, 8, 10). One would hope to obtain linear estimates for $N$ in $n$, which, for example, has been achieved by J. Bourgain and L. Tzafriri in 3 for $X_{n}=\ell_{n}^{p}, 1 \leq p \leq \infty$. However, for many other Banach spaces, the best known estimates are often super-exponential.

For instance, P. F. X. Müller showed that for $X_{d_{n}}=H_{n}^{1}, X_{d_{n}}=\left(H_{n}^{1}\right)^{*}$ (see [12]) and $X_{d_{n}}=L^{p}, 1<p<\infty$ (see [14]), where $d_{n}=2^{n+1}-1$, the estimate for $N$ is a

Date: November 9, 2018.

2010 Mathematics Subject Classification. 46B07, 30H10, 46B25, 60G46.

Key words and phrases. Factorization, local theory, classical Banach spaces, Hardy spaces, $\mathrm{BMO}, S L^{\infty}$

Supported by the Austrian Science Foundation (FWF) Pr.Nr. P28352. 
nested exponential, e.g.

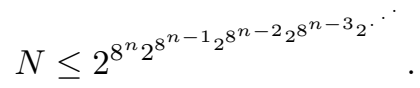

Another example where $N$ is estimated by a nested exponential in $n$, is the one parameter space $X_{d_{n}}=S L_{n}^{\infty}$ (see [10]); a similar statement is true for the biparameter mixed norm Hardy spaces $H_{n}^{p}\left(H_{n}^{q}\right), 1 \leq p, q<\infty$ and their duals (see [8]).

The cause for the super-exponential growth in the previous three examples can be pinpointed exactly: the use of combinatorics. In this work, we introduce a new method, which replaces these combinatorics with an entirely probabilistic approach. Consequently, we obtain for $X_{d_{n}}=H_{n}^{p}, X_{d_{n}}=\left(H_{n}^{p}\right)^{*}, 1 \leq p<\infty$ and $X_{d_{n}}=S L_{n}^{\infty}$ (see Theorem 3.1) the estimate

$$
N \leq c n, \quad \text { where } c=c(\delta, \Gamma, \eta)>0 .
$$

\section{Notation}

The collection of dyadic intervals $\mathcal{D}$ contained in the unit interval $[0,1)$ is given by

$$
\mathcal{D}=\left\{\left[(k-1) 2^{-n}, k 2^{-n}\right): n \in \mathbb{N}_{0}, 1 \leq k \leq 2^{n}\right\} .
$$

Let $|\cdot|$ denote the Lebesgue measure. For any $N \in \mathbb{N}_{0}$ we put

$$
\mathcal{D}_{N}=\left\{I \in \mathcal{D}:|I|=2^{-N}\right\} \quad \text { and } \quad \mathcal{D}_{\leq N}=\bigcup_{n=0}^{N} \mathcal{D}_{n} .
$$

Given $n \in \mathbb{N}_{0}$ and a dyadic interval $I \in \mathcal{D}_{n}$, we define $I^{-}, I^{+} \in \mathcal{D}_{n+1}$ by

$$
I^{+} \cup I^{-}=I \quad \text { and } \quad \inf I^{+}<\inf I^{-} .
$$

The $L^{\infty}$-normalized Haar system $h_{I}, I \in \mathcal{D}$ is given by

$$
h_{I}=\chi_{I^{+}}-\chi_{I^{-}}, \quad I \in \mathcal{D},
$$

where $\chi_{A}$ denotes the characteristic function of a set $A \subset[0,1)$.

Given $1 \leq p<\infty$, the Hardy space $H^{p}$ is the completion of

$$
\operatorname{span}\left\{h_{I}: I \in \mathcal{D}\right\}
$$

under the square function norm

$$
\left\|\sum_{I \in \mathcal{D}} a_{I} h_{I}\right\|_{H^{p}}=\left(\int_{0}^{1}\left(\sum_{I \in \mathcal{D}} a_{I}^{2} h_{I}^{2}(x)\right)^{p / 2} \mathrm{~d} x\right)^{1 / p} .
$$

For each $n \in \mathbb{N}_{0}$, we define the finite dimensional space

$$
H_{n}^{p}=\operatorname{span}\left\{h_{I}: I \in \mathcal{D}_{\leq n}\right\} \subset H^{p} .
$$

The non-separable Banach space $S L^{\infty}$ is given by

$$
S L^{\infty}=\left\{f \in L^{2}:\|f\|_{S L^{\infty}}<\infty\right\},
$$

equipped with the norm

$$
\left\|\sum_{I \in \mathcal{D}} a_{I} h_{I}\right\|_{S L^{\infty}}=\left\|\left(\sum_{I \in \mathcal{D}} a_{I}^{2} h_{I}^{2}\right)^{1 / 2}\right\|_{L^{\infty}} .
$$

For all $n \in \mathbb{N}_{0}$, we define the finite dimensional space

$$
S L_{n}^{\infty}=\operatorname{span}\left\{h_{I}: I \in \mathcal{D}_{\leq n}\right\} \subset S L^{\infty} .
$$

We define the duality pairing $\langle\cdot, \cdot\rangle: S L^{\infty} \times H^{1} \rightarrow \mathbb{R}$ by

$$
\langle f, g\rangle=\int_{0}^{1} f(x) g(x) \mathrm{d} x, \quad f \in S L^{\infty}, g \in H^{1} .
$$


An elementary computation (see e.g. [5]) shows that

$$
|\langle f, g\rangle| \leq\|f\|_{S L^{\infty}}\|g\|_{H^{1}}, \quad f \in S L^{\infty}, g \in H^{1} .
$$

\section{MAIN RESUlt}

Let $1 \leq p<\infty$ and recall that we put $d_{n}=2^{n+1}-1, n \in \mathbb{N}$. Our main result Theorem 3.1 gives a quantitative estimate for $N=N(n, \delta, \Gamma, \eta)$ in Question 1.1 for the spaces $W_{d_{n}}=H_{n}^{p}, W_{d_{n}}=\left(H_{n}^{p}\right)^{*}$ and $W_{d_{n}}=S L_{n}^{\infty}$.

Theorem 3.1. Let $1 \leq p<\infty$, and let $\left(W_{k}: k \in \mathbb{N}\right)$ denote one of the following three sequences of spaces:

$$
\left(H_{k}^{p}: k \in \mathbb{N}\right), \quad\left(\left(H_{k}^{p}\right)^{*}: k \in \mathbb{N}\right), \quad\left(S L_{k}^{\infty}: k \in \mathbb{N}\right) .
$$

Let $n \in \mathbb{N}$ and $\delta>0, \Gamma, \eta>0$. Define the integer $N=N(n, \delta, \Gamma, \eta)$ by the formula

$$
N=19(n+2)+\left\lfloor 4 \log _{2}(\Gamma / \delta)+4 \log _{2}\left(1+\eta^{-1}\right)\right\rfloor .
$$

Then for any operator $T: W_{N} \rightarrow W_{N}$ satisfying

$$
\|T\| \leq \Gamma \quad \text { and } \quad\left|\left\langle T h_{K}, h_{K}\right\rangle\right| \geq \delta|K|, \quad K \in \mathcal{D}_{\leq N},
$$

there exist bounded linear operators $E: W_{n} \rightarrow W_{N}$ and $F: W_{N} \rightarrow W_{n}$, such that the diagram

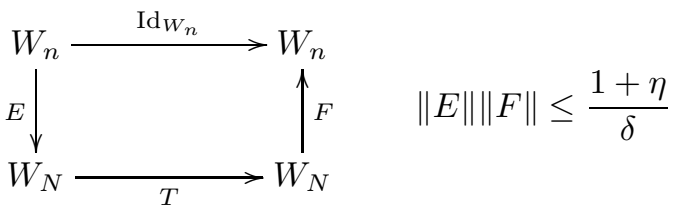

is commutative.

Firstly, we remark that the linear dependence of $N$ on $n$ amounts to a polynomial dependence of the dimensions of the respective spaces; i.e. $\operatorname{dim} W_{N}$ is a polynomial in $\operatorname{dim} W_{n}$.

Secondly, although very similar in spirit, since the results of [3] concern operators with large diagonal with respect to the standard unit vector basis in $\ell_{n}^{p}$, the results in 3 are not applicable in the context of Theorem 3.1, which is concerned with operators having large diagonal with respect to the Haar system.

Thirdly, the novelty of Theorem [3.1] is the above formula (3.2) for $N$, specifically the linear relation between $N$ and $n$. Indeed, we point out that for the previous results

$$
\begin{aligned}
& \triangleright\left(W_{k}: k \in \mathbb{N}\right)=\left(H_{k}^{1}: k \in \mathbb{N}\right) \text { and }\left(W_{k}: k \in \mathbb{N}\right)=\left(\left(H_{k}^{1}\right)^{*}: k \in \mathbb{N}\right) \text { in [12], } \\
& \triangleright\left(W_{k}: k \in \mathbb{N}\right)=\left(H_{k}^{p}: k \in \mathbb{N}\right), 1<p<\infty \text { in [14], } \\
& \triangleright\left(W_{k}: k \in \mathbb{N}\right)=\left(S L_{k}^{\infty}: k \in \mathbb{N}\right) \text { in [10], }
\end{aligned}
$$

the relation between $N$ and $n$ is super-exponential. The cause for this growth is the use of combinatorics. In a first step, these combinatorial methods are used to almost diagonalize the operator $T$, and then, in a second step, probabilistic arguments are employed to preserve the large diagonal of $T$.

By contrast, our new and entirely probabilistic approach almost diagonalizes $T$ and preserves its large diagonal in a single step (see Section 4).

\section{RANDOM BLOCK BASES}

Given $1 \leq p<\infty$, let $W_{N}$ denote either $W_{N}=H^{p}, W_{N}=\left(H^{p}\right)^{*}$ or $W_{N}=S L_{N}^{\infty}$. In this section, we will show that every operator $T: W_{N} \rightarrow W_{N}$ is almost diagonalized by random block bases $\theta \mapsto b_{I}^{(\theta)} \subset W_{N}, I \in \mathcal{D}_{\leq n}$. 
To this end, let $\mathbb{P}$ denote the uniform measure on $\{ \pm 1\}^{\mathcal{D}}$, and let $\mathbb{E}$ denote the expectation with respect to the probability measures $\mathbb{P}$. Given $n, N \in \mathbb{N}$ and pairwise disjoint sets $\mathcal{B}_{I} \subset \mathcal{D}_{\leq N}, I \in \mathcal{D}_{\leq n}$, we define the random block basis

$$
b_{I}^{(\theta)}=\sum_{K \in \mathcal{B}_{I}} \theta_{K} h_{K}, \quad I \in \mathcal{D}_{\leq n}, \theta \in\{ \pm 1\} .
$$

Given a linear operator $T: W_{N} \rightarrow W_{N}$, we define the random variables $Y_{I, I^{\prime}}, Z_{I}$ by putting

$$
\begin{aligned}
Y_{I, I^{\prime}}(\theta) & =\left\langle T b_{I}^{(\theta)}, b_{I^{\prime}}^{(\theta)}\right\rangle, & I, I^{\prime} \in \mathcal{D}_{\leq n}, I \neq I^{\prime}, \theta \in\{ \pm 1\} \\
Z_{I}(\theta) & =\left\langle T b_{I}^{(\theta)}, b_{I}^{(\theta)}\right\rangle-\sum_{K \in \mathcal{B}_{I}}\left\langle T h_{K}, h_{K}\right\rangle, & I \in \mathcal{D}_{\leq n}, \theta \in\{ \pm 1\}
\end{aligned}
$$

The following Theorem 4.1 asserts that the matrix-valued random variable $\theta \mapsto$ $\left(\left\langle T b_{I}^{(\theta)}, b_{I^{\prime}}^{(\theta)}\right\rangle\right)_{I, I^{\prime} \in \mathcal{D}_{\leq n}}$ is for the most part (depending on the collections $\mathcal{B}_{I}, I \in$ $\left.\mathcal{D}_{\leq n}\right)$ centered around the diagonal matrix $\operatorname{diag}\left(\sum_{K \in \mathcal{B}_{I}}\left\langle T h_{K}, h_{K}\right\rangle\right)_{I \in \mathcal{D}_{\leq n}}$.

Theorem 4.1. Let $n, N \in \mathbb{N}$, and let $\mathcal{B}_{I} \subset \mathcal{D}_{\leq N}, I \in \mathcal{D}_{\leq n}$ denote non-empty collections of dyadic intervals satisfying

$$
\begin{aligned}
\mathcal{B}_{I} \cap \mathcal{B}_{I^{\prime}} & =\emptyset, & & I, I^{\prime} \in \mathcal{D}_{\leq n}, I \neq I^{\prime} \\
K \cap K^{\prime} & =\emptyset, & & K, K^{\prime} \in \mathcal{B}_{I}, K \neq K^{\prime}, I \in \mathcal{D}_{\leq n} .
\end{aligned}
$$

Define $\alpha$ by putting

$$
\alpha=\max \left\{|K|: K \in \mathcal{B}_{I}, I \in \mathcal{D}_{\leq n}\right\} .
$$

Given $1 \leq p<\infty$, let $W_{N}$ denote either $W_{N}=H^{p}, W_{N}=\left(H^{p}\right)^{*}$ or $W_{N}=S L_{N}^{\infty}$. Then for any operator $T: W_{N} \rightarrow W_{N}$, we have that

$$
\mathbb{E} Y_{I, I^{\prime}}=\mathbb{E} Z_{I}=0, \quad I, I^{\prime} \in \mathcal{D}, I \neq I^{\prime},
$$

and the random variables $Y_{I, I^{\prime}}, Z_{I}$ satisfy the estimates

$$
\mathbb{E} Y_{I, I^{\prime}}^{2} \leq\|T\|^{2} \alpha^{1 / 2}, \quad \mathbb{E} Z_{I}^{2} \leq 2\|T\|^{2} \alpha^{1 / 2},
$$

for all $I, I^{\prime} \in \mathcal{D}, I \neq I^{\prime}$.

Before we proceed to the proof of Theorem 4.1, we record the following elementary facts.

Lemma 4.2. Let $\mathcal{B}$ be a non-empty, finite collection of pairwise disjoint dyadic intervals, and define

$$
b^{(\theta)}=\sum_{K \in \mathcal{B}} \theta_{K} h_{K}, \quad \theta \in\{ \pm 1\}^{\mathcal{D}} .
$$

Then for all $1 \leq p<\infty, 1<p^{\prime} \leq \infty$ with $\frac{1}{p}+\frac{1}{p^{\prime}}=1$, we have

$$
\left\|b^{(\theta)}\right\|_{H^{p}}=|\bigcup \mathcal{B}|^{1 / p}, \quad\left\|b^{(\theta)}\right\|_{\left(H^{p}\right)^{*}}=|\bigcup \mathcal{B}|^{1 / p^{\prime}} \quad \text { and } \quad\left\|b^{(\theta)}\right\|_{S L^{\infty}}=1 .
$$

Proof of Lemma 4.2. The proof is simple and straightforward, and therefore omitted.

Proof of Theorem 4.1. Clearly, $\mathbb{E} Y_{I, I^{\prime}}=\mathbb{E} Z_{I}=0$.

Note that for $K_{0}, K_{1}, K_{0}^{\prime}, K_{1}^{\prime} \in \mathcal{D}$, we have $\mathbb{E} \theta_{K_{0}} \theta_{K_{1}} \theta_{K_{0}^{\prime}} \theta_{K_{1}^{\prime}}=1$ if and only if one of the following conditions is satisfied:

(K1) $K_{0}=K_{1}=K_{0}^{\prime}=K_{1}^{\prime}$;

(K2) $K_{0}=K_{1} \neq K_{0}^{\prime}=K_{1}^{\prime}$; 
DIMENSION DEPENDENCE OF FACTORIZATION PROBLEMS: HARDY SPACES AND $S L_{n}^{\infty} 5$

(K3) $K_{0}=K_{0}^{\prime} \neq K_{1}=K_{1}^{\prime}$;

(K4) $K_{0}=K_{1}^{\prime} \neq K_{1}=K_{0}^{\prime}$.

Estimates For $Y_{I, I^{\prime}}$, IF $W_{N}=H_{N}^{p}$. Note that

$$
\mathbb{E} Y_{I, I^{\prime}}^{2}(\theta)=\sum_{\substack{K_{0}, K_{1} \in \mathcal{B}_{I} \\ K_{0}^{\prime}, K_{1}^{\prime} \in \mathcal{B}_{I^{\prime}}}} \mathbb{E} \theta_{K_{0}} \theta_{K_{1}} \theta_{K_{0}^{\prime}} \theta_{K_{1}^{\prime}}\left\langle T h_{K_{0}}, h_{K_{0}^{\prime}}\right\rangle\left\langle T h_{K_{1}}, h_{K_{1}^{\prime}}\right\rangle .
$$

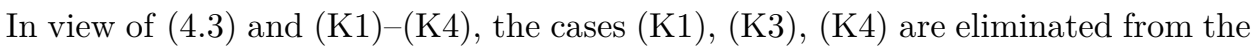
sum in (4.9). Thus, with only (K2) terms left, (4.9) reads as follows:

$$
\mathbb{E} Y_{I, I^{\prime}}^{2}=\sum_{\substack{K_{0} \in \mathcal{B}_{I} \\ K_{0}^{\prime} \in \mathcal{B}_{I^{\prime}}}}\left\langle T h_{K_{0}}, h_{K_{0}^{\prime}}\right\rangle^{2} .
$$

Put $a_{K_{0}, K_{0}^{\prime}}=\left\langle T h_{K_{0}}, h_{K_{0}^{\prime}}\right\rangle$ and note that

$$
\left|a_{K_{0}, K_{0}^{\prime}}\right| \leq\|T\|\left|K_{0}\right|^{1 / p}\left|K_{0}^{\prime}\right|^{1 / p^{\prime}}
$$

We will now estimate (4.10) in two different ways.

Firstly, we rewrite (4.10) and then use duality to obtain

$$
\begin{aligned}
\mathbb{E} Y_{I, I^{\prime}}^{2} & =\sum_{K_{0} \in \mathcal{B}_{I}}\left\langle T h_{K_{0}}, \sum_{K_{0}^{\prime} \in \mathcal{B}_{I^{\prime}}} a_{K_{0}, K_{0}^{\prime}} h_{K_{0}^{\prime}}\right\rangle \\
& \leq\left.\sum_{K_{0} \in \mathcal{B}_{I}}\|T\||| K_{0}\right|^{1 / p}\left\|\sum_{K_{0}^{\prime} \in \mathcal{B}_{I^{\prime}}} a_{K_{0}, K_{0}^{\prime}} h_{K_{0}^{\prime}}\right\|_{\left(H^{p}\right)^{*}} .
\end{aligned}
$$

(4.3), Lemma 4.2 and (4.11) give us

$$
\begin{aligned}
\mathbb{E} Y_{I, I^{\prime}}^{2} & \leq \sum_{K_{0} \in \mathcal{B}_{I}}\|T\|\left|K_{0}\right|^{1 / p} \max _{K_{0}^{\prime} \in \mathcal{B}_{I^{\prime}}}\left|a_{K_{0}, K_{0}^{\prime}}\right|\left\|\sum_{K_{0}^{\prime} \in \mathcal{B}_{I^{\prime}}} h_{K_{0}^{\prime}}\right\| \\
& \leq \sum_{K_{0} \in \mathcal{B}_{I}}\|T\|^{2}\left|K_{0}\right|^{2 / p} \max _{K_{0}^{\prime} \in \mathcal{B}_{I^{\prime}}}\left|K_{0}^{\prime}\right|^{1 / p^{\prime}} .
\end{aligned}
$$

Applying Hölder's inequality yields

$$
\mathbb{E} Y_{I, I^{\prime}}^{2} \leq\|T\|^{2} \max _{\substack{K_{0} \in \mathcal{B}_{I} \\ K_{0}^{\prime} \in \mathcal{B}_{I^{\prime}}}}\left|K_{0}\right|^{2 / p-1}\left|K_{0}^{\prime}\right|^{1 / p^{\prime}}
$$

Using (4.4) gives us the estimate

$$
\mathbb{E} Y_{I, I^{\prime}}^{2} \leq\|T\|^{2} \alpha^{1 / p}
$$

Secondly, we rewrite (4.10) as follows:

$$
\mathbb{E} Y_{I, I^{\prime}}^{2}=\sum_{K_{0}^{\prime} \in \mathcal{B}_{I^{\prime}}}\left\langle T \sum_{K_{0} \in \mathcal{B}_{I}} a_{K_{0}, K_{0}^{\prime}} h_{K_{0}}, h_{K_{0}^{\prime}}\right\rangle
$$

The analogous computation to the one above shows

$$
\mathbb{E} Y_{I, I^{\prime}}^{2} \leq\|T\|^{2} \alpha^{1 / p^{\prime}} .
$$

Finally, combining (4.12) and (4.13) yields:

$$
\mathbb{E} Y_{I, I^{\prime}}^{2} \leq\|T\|^{2} \alpha^{1 / 2} \text {. }
$$

Estimates FOR $Z_{I}$, IF $W_{N}=H_{N}^{p}$. In the following sums, the variables $K_{0}, K_{0}^{\prime}, K_{1}, K_{1}^{\prime}$ will always be summed over the collection $\mathcal{B}_{I}$. Note that

$$
\mathbb{E} Z_{I}^{2}(\theta)=\sum_{\substack{K_{0} \neq K_{0}^{\prime} \\ K_{1} \neq K_{1}^{\prime}}} \mathbb{E} \theta_{K_{0}} \theta_{K_{1}} \theta_{K_{0}^{\prime}} \theta_{K_{1}^{\prime}}\left\langle T h_{K_{0}}, h_{K_{0}^{\prime}}\right\rangle\left\langle T h_{K_{1}}, h_{K_{1}^{\prime}}\right\rangle .
$$


In view of (4.3) and (K1)-(K4), the cases (1) and (K3), are eliminated from the sum in (4.15).

If we restrict the sum in (4.15) to Case (제), (4.15) reads

$$
\mathbb{E} Z_{I}^{2}(\theta)=\sum_{K_{0} \neq K_{0}^{\prime}}\left\langle T h_{K_{0}}, h_{K_{0}^{\prime}}\right\rangle^{2} .
$$

Note that the expressions (4.10) and (4.16) are algebraically the same, except for the conditions $I \neq I^{\prime}$ in (4.10) and $I=I^{\prime}$ in (4.16). Hence, we can repeat the proof for $Y_{I, I^{\prime}}$, which yields

$$
\mathbb{E} Z_{I}^{2} \leq\|T\|^{2} \alpha^{1 / 2}
$$

Restricting the sum in (4.15) to Case (K4) gives us

$$
\mathbb{E} Z_{I}^{2}(\theta)=\sum_{K_{0} \neq K_{1}}\left\langle T h_{K_{0}}, h_{K_{1}}\right\rangle\left\langle T h_{K_{1}}, h_{K_{0}}\right\rangle .
$$

Put $a_{K_{0}, K_{1}}=\left\langle T h_{K_{0}}, h_{K_{1}}\right\rangle$ and note that

$$
\left|a_{K_{0}, K_{1}}\right| \leq\|T\|\left|K_{0}\right|^{1 / p}\left|K_{1}\right|^{1 / p^{\prime}} .
$$

We will now estimate (4.18) in two different ways. Firstly, rewriting (4.18) and then using duality yields

$$
\mathbb{E} Z_{I}^{2}=\sum_{K_{0}}\left\langle T \sum_{K_{1}} a_{K_{0}, K_{1}} h_{K_{1}}, h_{K_{0}}\right\rangle \leq \sum_{K_{0}}\|T\|\left\|\sum_{K_{1}} a_{K_{0}, K_{1}} h_{K_{1}}\right\|_{H^{p}}\left|K_{0}\right|^{1 / p^{\prime}} .
$$

(4.3), Lemma 4.2 and (4.19) give us

$$
\begin{aligned}
\mathbb{E} Z_{I}^{2} & \leq \sum_{K_{0}}\|T\| \max _{K_{1}}\left|a_{K_{0}, K_{1}}\right|\left\|\sum_{K_{1}} h_{K_{1}}\right\|{ }_{H^{p}}\left|K_{0}\right|^{1 / p^{\prime}} \\
& \leq \sum_{K_{0}}\|T\|^{2}\left|K_{0}\right| \max _{K_{1}}\left|K_{1}\right|^{1 / p^{\prime}}=\|T\|^{2} \max _{K_{1}}\left|K_{1}\right|^{1 / p^{\prime}} .
\end{aligned}
$$

Using (4.4), we obtain the estimate

$$
\mathbb{E} Z_{I}^{2} \leq\|T\|^{2} \alpha^{1 / p^{\prime}}
$$

Secondly, we rewrite (4.18) as follows:

$$
\mathbb{E} Z_{I}^{2}=\sum_{K_{1}}\left\langle T h_{K_{1}}, \sum_{K_{0}} a_{K_{0}, K_{1}} h_{K_{0}}\right\rangle .
$$

The analogous computation to the one above shows

$$
\mathbb{E} Z_{I}^{2} \leq\|T\|^{2} \alpha^{1 / p} .
$$

Finally, combining (4.20) with (4.21) gives us

$$
\mathbb{E} Z_{I}^{2} \leq\|T\|^{2} \alpha^{1 / 2} .
$$

in Case (K4).

Adding (4.17) and (4.22) yields

$$
\mathbb{E} Z_{I}^{2} \leq 2\|T\|^{2} \alpha^{1 / 2} .
$$

Estimates For $W_{N}=\left(H_{N}^{p}\right)^{*}$ AND $W_{N}=S L_{N}^{\infty}$. If $W_{N}=\left(H_{N}^{p}\right)^{*}$, we repeat the above proof, but with the roles of $H_{N}^{p}$ and $\left(H_{N}^{p}\right)^{*}$ reversed.

If $W_{N}=S L_{N}^{\infty}$, we only need to repeat half of the above proof (only the parts where the inner sum is on the $S L^{\infty}$ side of the duality pairing). To be more precise, we repeat the proof for estimate (4.13) for $Y_{I, I^{\prime}}$, and the proof for the 
estimates (4.17) (which is actually repeating the proof for $Y_{I, I^{\prime}}$, again) and (4.20) for $Z_{I}$. This way, we obtain the estimates

$$
\mathbb{E} Y_{I, I^{\prime}}^{2} \leq\|T\|^{2} \alpha \quad \text { and } \quad \mathbb{E} Z_{I}^{2} \leq 2\|T\|^{2} \alpha .
$$

\section{EMBEDDINGS, PROJECTIONS AND FACTORIZATION}

First, we record essential facts about embeddings and projections in $H^{p},\left(H^{p}\right)^{*}$, $1 \leq p<\infty$ and $S L^{\infty}$, and then we prove the main result Theorem 3.1.

5.1. Jones' compatibility condition. Given $\mathcal{B}_{I} \subset \mathcal{D}, I \in \mathcal{D}$, we put $B_{I}=\bigcup \mathcal{B}_{I}$. We say that the collections $\mathcal{B}_{I}, I \in \mathcal{D}$ satisfy Jones' compatibility condition (C) (see [7; see also [13]) with constant $\kappa \geq 1$, if the following four conditions are satisfied:

(C1) For each $I \in \mathcal{D}$, the collection $\mathcal{B}_{I}$ consists of finitely many pairwise disjoint dyadic intervals; moreover, $\mathcal{B}_{I} \cap \mathcal{B}_{I^{\prime}}=\emptyset$, whenever $I, I^{\prime} \in \mathcal{D}, I \neq I^{\prime}$.

(C2) For every $I \in \mathcal{D}$, we have that $B_{I^{-}} \cup B_{I^{+}} \subset B_{I}$ and $B_{I^{-}} \cap B_{I^{+}}=\emptyset$.

(C3) $\kappa^{-1}|I| \leq\left|B_{I}\right| \leq \kappa|I|$, for all $I \in \mathcal{D}$.

(C4) For all $I_{0}, I \in \mathcal{D}$ with $I_{0} \subset I$ and $K \in \mathcal{B}_{I}$, we have $\frac{\left|K \cap B_{I_{0}}\right|}{|K|} \geq \kappa^{-1} \frac{\left|B_{I_{0}}\right|}{\left|B_{I}\right|}$.

Theorem 5.1. Let $\mathcal{B}_{I} \subset \mathcal{D}, I \in \mathcal{D}$ satisfy Jones' compatibility condition (C) with constant $\kappa=1$. Let $\theta \in\{ \pm 1\}^{\mathcal{D}}$ and define

$$
b_{I}^{(\theta)}=\sum_{K \in \mathcal{B}_{I}} \theta_{K} h_{K}, \quad I \in \mathcal{D} .
$$

Given $1 \leq p<\infty$, let $W$ denote either $H^{p},\left(H^{p}\right)^{*}$ or $S L^{\infty}$. Then the operators $B^{(\theta)}, A^{(\theta)}: W \rightarrow W$ given by

$$
B^{(\theta)} f=\sum_{I \in \mathcal{D}} \frac{\left\langle f, h_{I}\right\rangle}{\left\|h_{I}\right\|_{2}^{2}} b_{I}^{(\theta)} \quad \text { and } \quad A^{(\theta)} f=\sum_{I \in \mathcal{D}} \frac{\left\langle f, b_{I}^{(\theta)}\right\rangle}{\left\|b_{I}^{(\theta)}\right\|_{2}^{2}} h_{I}
$$

satisfy the estimates

$$
\begin{aligned}
& \left\|B^{(\theta)} f\right\|_{W} \leq\|f\|_{W}, \quad f \in W, \\
& \left\|A^{(\theta)} f\right\|_{W} \leq\|f\|_{W}, \quad f \in W .
\end{aligned}
$$

Moreover, the diagram

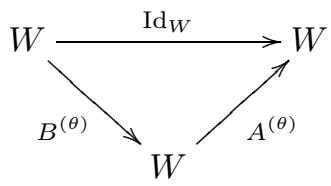

is commutative and the composition $P^{(\theta)}=B^{(\theta)} A^{(\theta)}$ is the norm 1 projection $P^{(\theta)}$ : $W \rightarrow W$ given by

$$
P^{(\theta)}(f)=\sum_{I \in \mathcal{D}} \frac{\left\langle f, b_{I}^{(\theta)}\right\rangle}{\left\|b_{I}\right\|_{2}^{2}} b_{I}^{(\theta)}
$$

Consequently, the range of $B^{(\theta)}$ is complemented (by $\left.P^{(\theta)}\right)$, and $B^{(\theta)}$ is an isometric isomorphism onto its range.

Remark 5.2. In 4, Gamlen and Gaudet showed a similar version of Theorem 5.1 for $W=L^{p}, 1<p<\infty$. Let us point out two major aspects of their method: Firstly, they are using functions $\left(d_{i}\right)_{i=1}^{\infty}$, which are not adapted to any dyadic filtration, therefore, their method is not applicable in $H^{p}, 1<p<\infty$. Secondly, condition (C4) is not part of their hypothesis. Instead, the collections $\mathcal{B}_{I}, I \in \mathcal{D}$ and the sets $\left\{b_{I}^{(\theta)}= \pm 1\right\}, I \in \mathcal{D}$ are linked, so that their projection $P$ can be viewed as a conditional expectation. Hence, $P$ is bounded in $L^{1}$ and their result can be extended to $L^{1}$. 
In 6. Proposition 9.6], Johnson, Maurey, Schechtman and Tzafriri specify conditions for a block basis of the Haar system, so that the conclusion of Theorem [5.1 is true for $W=H^{p}, 1<p<\infty$. Since the proof relies on Stein's martingale inequality, their result does not extend to $W=H^{1}$ or $W=\left(H^{1}\right)^{*}$. If Jones' compatibility condition (C) is satisfied, the operator $B^{(\theta)}$ and the projection $P^{(\theta)}$ in Theorem 5.1 are the same as the respective operators occurring in [6, Proposition 9.6].

In [7, Jones showed Theorem 5.1 for $W=H^{1}$ and $W=\left(H^{1}\right)^{*}$. In order to achieve this, it was crucial to have condition (C4) in place.

The case $W=S L^{\infty}$ is proved in [9], even without requiring (C3).

5.2. Proof of the main result Theorem 3.1. For convenience, we repeat Theorem 3.1 here.

Theorem 5.3 (Main result Theorem 3.1). Let $1 \leq p<\infty$, and let $\left(W_{k}: k \in \mathbb{N}\right)$ denote one of the following three sequences of spaces:

$$
\left(H_{k}^{p}: k \in \mathbb{N}\right), \quad\left(\left(H_{k}^{p}\right)^{*}: k \in \mathbb{N}\right), \quad\left(S L_{k}^{\infty}: k \in \mathbb{N}\right) .
$$

Let $n \in \mathbb{N}$ and $\delta, \Gamma, \eta>0$. Define the integer $N=N(n, \delta, \Gamma, \eta)$ by the formula

$$
N=19(n+2)+\left\lfloor 4 \log _{2}(\Gamma / \delta)+4 \log _{2}\left(1+\eta^{-1}\right)\right\rfloor .
$$

Then for any operator $T: W_{N} \rightarrow W_{N}$ satisfying

$$
\|T\| \leq \Gamma \quad \text { and } \quad\left|\left\langle T h_{K}, h_{K}\right\rangle\right| \geq \delta|K|, \quad K \in \mathcal{D}_{\leq N},
$$

there exist bounded linear operators $E: W_{n} \rightarrow W_{N}$ and $F: W_{N} \rightarrow W_{n}$, such that the diagram

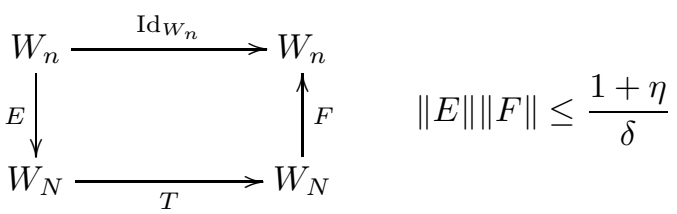

is commutative.

Proof. Define the norm 1 multiplication operator $M: W_{N} \rightarrow W_{N}$ as the linear extension of

$$
h_{K} \mapsto \operatorname{sign}\left(\left\langle T h_{K}, h_{K}\right\rangle\right) h_{K}, \quad K \in \mathcal{D}_{\leq N},
$$

and observe that by (5.8), we obtain

$$
\left\langle T M h_{K}, h_{K}\right\rangle=\left|\left\langle T h_{K}, h_{K}\right\rangle\right| \geq \delta|K|, \quad K \in \mathcal{D}_{\leq N} .
$$

Thus, we can assume that

$$
\left\langle T h_{K}, h_{K}\right\rangle \geq \delta|K|, \quad K \in \mathcal{D}_{\leq N} .
$$

Before we proceed, we define the following two constants: Let $m_{0} \in \mathbb{N}_{0}$ be the smallest integer for which

$$
2^{m_{0}}>\frac{2^{6(n+2)} \Gamma^{4}}{\eta_{0}^{4}}, \quad \text { where } \quad \eta_{0}=\frac{\eta \delta}{(1+\eta) 2^{3(n+2)}} .
$$

Step 1: Overview. The operators $E$ and $F$ will be defined in terms of a block basis $b_{I}^{(\theta)}, I \in \mathcal{D}_{\leq n}$ of the Haar system $h_{K}, K \in \mathcal{D}_{\leq N}$ having the following form:

$$
b_{I}^{(\theta)}=\sum_{K \in \mathcal{B}_{I}} \theta_{K} h_{K}, \quad I \in \mathcal{D}_{\leq n}, \theta \in\{ \pm 1\}^{\mathcal{D}} .
$$


Our goal is to find collections $\mathcal{B}_{I} \subset \mathcal{D}_{\leq N}, I \in \mathcal{D}_{\leq n}$ satisfying Jones' compatibility condition (C) with constant $\kappa=1$, and signs $\theta \in\{ \pm 1\}^{\mathcal{D}}$ such that

$$
\begin{aligned}
\left|\left\langle T b_{I}^{(\theta)}, b_{I^{\prime}}^{(\theta)}\right\rangle\right| & \leq \eta_{0}, & I, I^{\prime} \in \mathcal{D}_{\leq n}, I \neq I^{\prime}, \\
\left\langle T b_{I}^{(\theta)}, b_{I}^{(\theta)}\right\rangle & \geq\left(\delta-2^{n} \eta_{0}\right)\left\|b_{I}^{(\theta)}\right\|_{2}^{2}, & I \in \mathcal{D}_{\leq n} .
\end{aligned}
$$

Step 2: Constructing the RAndom Block basis $b_{I}^{(\theta)}, I \in \mathcal{D}_{\leq n}$. First, we will use a minimalist Gamlen-Gaudet construction to define the collections $\mathcal{B}_{I}$, $I \in \mathcal{D}_{\leq n}$, and then we will rely on Theorem 4.1 to find signs $\theta \in\{ \pm 1\}^{\mathcal{D}}$ such that (5.13) is satisfied.

We will now inductively define the collections $\mathcal{B}_{I}, I \in \mathcal{D}_{\leq n}$. We begin by putting,

$$
\mathcal{B}_{[0,1)}=\mathcal{D}_{m_{0}} .
$$

Let $0 \leq k \leq n-1$ and assume that we have already constructed the collections $\mathcal{B}_{I}$, $I \in \mathcal{D}_{\leq k}$. Then, we define

$$
\mathcal{B}_{I^{+}}=\left\{K^{+}: K \in \mathcal{B}_{I}\right\} \quad \text { and } \quad \mathcal{B}_{I^{-}}=\left\{K^{-}: K \in \mathcal{B}_{I}\right\}, \quad I \in \mathcal{D}_{k} .
$$

One can easily verify that the collections, $\mathcal{B}_{I}, I \in \mathcal{D}_{\leq n}$ satisfy Jones' compatibility condition (C) with constant $\kappa=1$.

Next, we will use a probabilistic argument to find $\theta \in\{ \pm 1\}^{\mathcal{D}}$ such that (5.13) is satisfied. To this end, let us now define the off-diagonal events

$$
O_{I, I^{\prime}}=\left\{\theta \in\{ \pm 1\}^{\mathcal{D}}:\left|\left\langle T b_{I}^{(\theta)}, b_{I^{\prime}}^{(\theta)}\right\rangle\right|>\eta_{0}\right\}, \quad I, I^{\prime} \in \mathcal{D}_{\leq n}, I \neq I^{\prime}
$$

and the diagonal events

$$
D_{I}=\left\{\theta \in\{ \pm 1\}^{\mathcal{D}}:\left|\left\langle T b_{I}^{(\theta)}, b_{I}^{(\theta)}\right\rangle-\sum_{K \in \mathcal{B}_{I}}\left\langle T h_{K}, h_{K}\right\rangle\right|>\eta_{0}\right\}, \quad I \in \mathcal{D}_{\leq n}
$$

By Theorem 4.1 and the definition of the random variables $Y_{I, I^{\prime}}, Z_{I}$ (see (4.2)), we obtain

$$
\mathbb{P}\left(O_{I, I^{\prime}}\right) \leq \frac{\Gamma^{2}}{2^{m_{0} / 2} \eta_{0}^{2}} \quad \text { and } \quad \mathbb{P}\left(D_{I}\right) \leq \frac{2 \Gamma^{2}}{2^{m_{0} / 2} \eta_{0}^{2}}, \quad I, I^{\prime} \in \mathcal{D}_{\leq n}, I \neq I .
$$

Using (5.17) and (5.11) gives us

$$
\mathbb{P}\left(\bigcup_{\substack{I, I^{\prime} \in \mathcal{D} \leq n \\ I \neq I^{\prime}}} O_{I, I^{\prime}} \cup \bigcup_{I \in \mathcal{D} \leq n} D_{I}\right) \leq \sum_{\substack{I, I^{\prime} \in \mathcal{D} \\ I \neq I^{\prime}}} \mathbb{P}\left(O_{I, I^{\prime}}\right)+\sum_{I \in \mathcal{D}_{\leq n}} \mathbb{P}\left(D_{I}\right) \leq \frac{2^{3(n+2)} \Gamma^{2}}{2^{m_{0} / 2} \eta_{0}^{2}}<1 .
$$

By (5.18) and the definition of the events $O_{I, I^{\prime}}, D_{I}$ (see (5.16)), we can find at least one $\theta \in\{ \pm 1\}^{\mathcal{D}}$ such that

$$
\begin{aligned}
\left|\left\langle T b_{I}^{(\theta)}, b_{I^{\prime}}^{(\theta)}\right\rangle\right| & \leq \eta_{0}, & I, I^{\prime} \in \mathcal{D}_{\leq n}, I \neq I^{\prime}, \\
\left|\left\langle T b_{I}^{(\theta)}, b_{I}^{(\theta)}\right\rangle-\sum_{K \in \mathcal{B}_{I}}\left\langle T h_{K}, h_{K}\right\rangle\right| & \leq \eta_{0}, & I \in \mathcal{D}_{\leq n} .
\end{aligned}
$$

Using (5.10), (C1), (C3) and that $\kappa=1$ by (5.15), we obtain

$$
\sum_{K \in \mathcal{B}_{I}}\left\langle T h_{K}, h_{K}\right\rangle \geq \sum_{K \in \mathcal{B}_{I}} \delta|K|=\delta\left|B_{I}\right|=\delta|I|, \quad I \in \mathcal{D}_{\leq n} .
$$

Combining this estimate with (5.19b) yields

$$
\left\langle T b_{I}^{(\theta)}, b_{I}^{(\theta)}\right\rangle \geq \delta|I|-\eta_{0}, \quad I \in \mathcal{D}_{\leq n} .
$$


By Lemma 4.2, we have $\left\|b_{I}^{(\theta)}\right\|_{2}^{2}=|I|$, thus, estimate (5.20) implies

$$
\left\langle T b_{I}^{(\theta)}, b_{I}^{(\theta)}\right\rangle \geq\left(\delta-\eta_{0} 2^{n}\right)\left\|b_{I}^{(\theta)}\right\|_{2}^{2}, \quad I \in \mathcal{D}_{\leq n} .
$$

Together, the estimates (5.19a) and (5.21) give us (5.13), that is

$$
\begin{aligned}
\mid\left\langle T b_{I}^{(\theta)}, b_{I^{\prime}}^{(\theta)}\right\rangle & \leq \eta_{0}, & I, I^{\prime} \in \mathcal{D}_{\leq n}, I \neq I^{\prime}, \\
\left\langle T b_{I}^{(\theta)}, b_{I}^{(\theta)}\right\rangle & \geq\left(\delta-2^{n} \eta_{0}\right)\left\|b_{I}^{(\theta)}\right\|_{2}^{2}, & I \in \mathcal{D}_{\leq n} .
\end{aligned}
$$

Step 3: Conclusion OF The PRoOF. By Theorem 5.1 the operators $B^{(\theta)}$ : $W_{n} \rightarrow W_{N}$ and $A^{(\theta)}: W_{N} \rightarrow W_{n}$ given by

$$
\begin{array}{ll}
B^{(\theta)} f=\sum_{I \in \mathcal{D}_{\leq n}} \frac{\left\langle f, h_{I}\right\rangle}{\left\|h_{I}\right\|_{2}^{2}} b_{I}^{(\theta)}, & f \in W_{n}, \\
A^{(\theta)} f=\sum_{I \in \mathcal{D}_{\leq n}} \frac{\left\langle f, b_{I}^{(\theta)}\right\rangle}{\left\|b_{I}^{(\theta)}\right\|_{2}^{2}} h_{I}, & f \in W_{N}
\end{array}
$$

satisfy the estimates

$$
\left\|B^{(\theta)}\right\| \leq 1 \quad \text { and } \quad\left\|A^{(\theta)}\right\| \leq 1 .
$$

The operator $P^{(\theta)}: W_{N} \rightarrow W_{N}$ defined by $P^{(\theta)}=B^{(\theta)} A^{(\theta)}$ is a norm 1 projection given by

$$
P^{(\theta)} f=\sum_{I \in \mathcal{D}_{\leq n}} \frac{\left\langle f, b_{I}^{(\theta)}\right\rangle}{\left\|b_{I}^{(\theta)}\right\|_{2}^{2}} b_{I}^{(\theta)}, \quad f \in W_{N} .
$$

Now, we define the subspace $Y$ by $Y=P^{(\theta)}\left(W_{N}\right)$, and note that the following diagram is commutative:

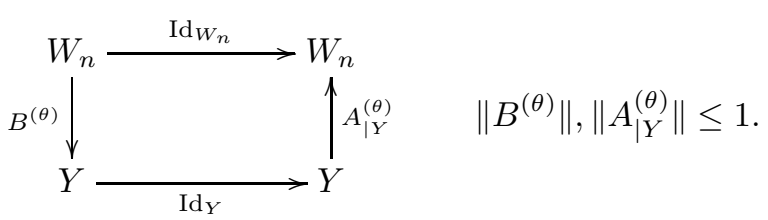

Next, we define $U^{(\theta)}: W_{N} \rightarrow Y$ by putting

$$
U^{(\theta)} f=\sum_{I \in \mathcal{D}_{\leq n}} \frac{\left\langle f, b_{I}^{(\theta)}\right\rangle}{\left\langle T b_{I}^{(\theta)}, b_{I}^{(\theta)}\right\rangle} b_{I}^{(\theta)}, \quad f \in W_{N} .
$$

By the 1-unconditionality of the Haar system, the definition of $P^{(\theta)}$ (see (5.25)) and the estimates (5.22), (5.24), we obtain

$$
\left\|U^{(\theta)}\right\| \leq \frac{\left\|P^{(\theta)}\right\|}{\delta-\eta_{0} 2^{n}} \leq \frac{1}{\delta-\eta_{0} 2^{n}} .
$$

Moreover, for all $g=\sum_{I \in \mathcal{D}_{\leq n}} a_{I} b_{I}^{(\theta)} \in Y$, we have the following identity:

$$
U^{(\theta)} T g-g=\sum_{\substack{I, I^{\prime} \in \mathcal{D}_{\leq n} \\ I \neq I^{\prime}}} a_{I^{\prime}} \frac{\left\langle T b_{I^{\prime}}^{(\theta)}, b_{I}^{(\theta)}\right\rangle}{\left\langle T b_{I}^{(\theta)}, b_{I}^{(\theta)}\right\rangle} b_{I}^{(\theta)}
$$

Note that Lemma 4.2 yields $\left|a_{I^{\prime}}\right| \leq \frac{\|g\|_{W_{N}}}{\left\|b_{I^{\prime}}^{(\theta}\right\|_{W_{N}}}$, thus we obtain from (15.22) that

$$
\left\|U^{(\theta)} T g-g\right\|_{W_{N}} \leq\left\|\sum_{\substack{I, I^{\prime} \in \mathcal{D} \leq n \\ I^{\prime} \neq I}} a_{I^{\prime}} \frac{\left\langle T b_{I^{\prime}}^{(\theta)}, b_{I}^{(\theta)}\right\rangle}{\left\langle T b_{I}^{(\theta)}, b_{I}^{(\theta)}\right\rangle} b_{I}^{(\theta)}\right\|_{W_{N}} \leq \frac{\eta_{0} 2^{3(n+1)}}{\delta-\eta_{0} 2^{n}}\|g\|_{W_{N}}
$$


Now, let $I: Y \rightarrow W_{N}$ denote the operator given by $I y=y$. By (5.11), we have that $\frac{\eta_{0} 2^{3(n+1)}}{\delta-\eta_{0} 2^{n}}<1$; hence (5.30) yields

$$
\left\|\left(U^{(\theta)} T I\right)^{-1} g\right\|_{W_{N}} \leq \frac{1}{1-\frac{\eta_{0} 2^{3(n+1)}}{\delta-\eta_{0} 2^{n}}}\|g\|_{W_{N}} .
$$

By (5.28), (5.31) and (5.11), the operator $V^{(\theta)}: W_{N} \rightarrow Y$ given by $V^{(\theta)}=$ $\left(U^{(\theta)} T I\right)^{-1} U^{(\theta)}$ satisfies the estimate

$$
\left\|V^{(\theta)}\right\| \leq \frac{1}{\delta-\eta_{0}\left(2^{n}+2^{3(n+1)}\right)} \leq \frac{1+\eta}{\delta}
$$

and the following diagram is commutative:

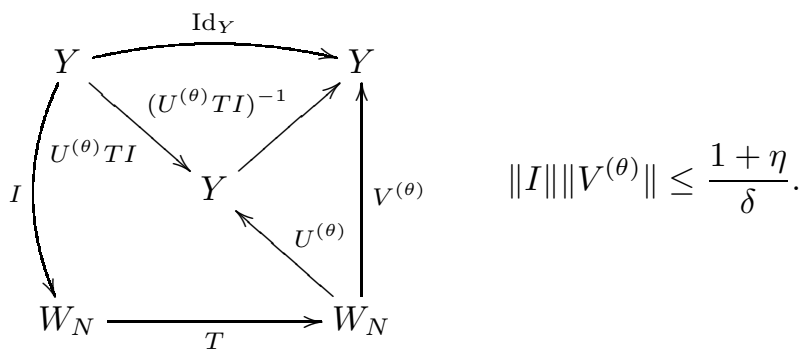

Merging the diagrams (5.26) and (5.32) yields

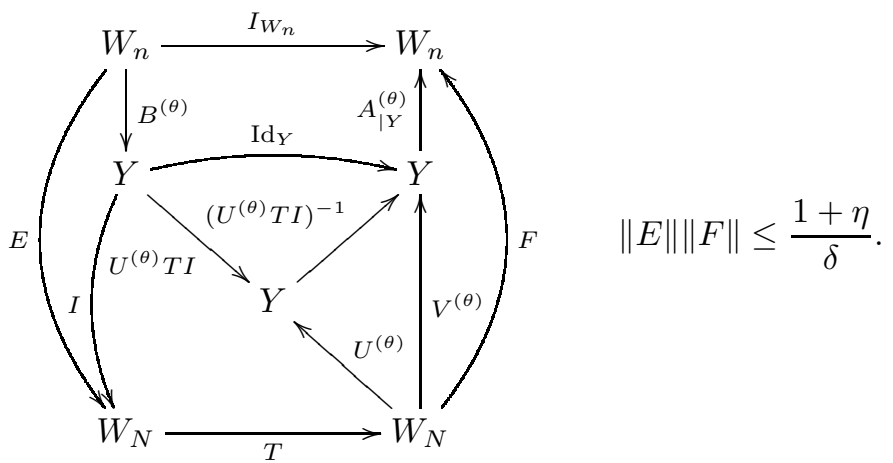

Finally, reviewing the construction of the block basis $b_{I}^{(\theta)}, I \in \mathcal{D}_{\leq n}$ (see (5.14) and (5.15) ) and the definitions of the operators involved in diagram (5.33), $N$ must be at least $m_{0}+n$; hence, considering the constants defined in (5.11) makes (5.7) an appropriate choice for $N$.

\section{Acknowledgments.}

It is my pleasure to thank P. F. X. Müller for many helpful discussions. Supported by the Austrian Science Foundation (FWF) Pr.Nr. P28352.

\section{REFERENCES}

[1] G. Blower. The Banach space $B\left(l^{2}\right)$ is primary. Bull. London Math. Soc., 22(2):176-182, 1990.

[2] J. Bourgain. On the primarity of $H^{\infty}$-spaces. Israel J. Math., 45(4):329-336, 1983.

[3] J. Bourgain and L. Tzafriri. Invertibility of "large" submatrices with applications to the geometry of Banach spaces and harmonic analysis. Israel J. Math., 57(2):137-224, 1987.

[4] J. L. B. Gamlen and R. J. Gaudet. On subsequences of the Haar system in $L_{p}[1,1](1 \leq p \leq$ $\infty)$. Israel J. Math., 15:404-413, 1973.

[5] A. M. Garsia. Martingale inequalities: Seminar notes on recent progress. W. A. Benjamin, Inc., Reading, Mass.-London-Amsterdam, 1973. Mathematics Lecture Notes Series.

[6] W. B. Johnson, B. Maurey, G. Schechtman, and L. Tzafriri. Symmetric structures in Banach spaces. Mem. Amer. Math. Soc., 19(217):v+298, 1979. 
[7] P. W. Jones. BMO and the Banach space approximation problem. Amer. J. Math., 107(4):853-893, 1985.

[8] R. Lechner. Factorization in mixed norm Hardy and BMO spaces. Studia Math., to appear. Preprint available on ArXiv.

[9] R. Lechner. Factorization in $S L^{\infty}$. Israel J. Math., to appear. Preprint available on ArXiv.

[10] R. Lechner. Direct sums of finite dimensional $S L_{n}^{\infty}$ spaces. ArXiv e-prints, Sept. 2017.

[11] R. Lechner and P. F. X. Müller. Localization and projections on bi-parameter BMO. Q. J. Math., 66(4):1069-1101, 2015.

[12] P. F. X. Müller. On projections in $H^{1}$ and BMO. Studia Math., 89(2):145-158, 1988.

[13] P. F. X. Müller. Isomorphisms between $H^{1}$ spaces, volume 66 of Instytut Matematyczny Polskiej Akademii Nauk. Monografie Matematyczne (New Series) [Mathematics Institute of the Polish Academy of Sciences. Mathematical Monographs (New Series)]. Birkhäuser Verlag, Basel, 2005.

[14] P. F. X. Müller. Two remarks on primary spaces. Math. Proc. Cambridge Philos. Soc., 153(3):505-523, 2012.

[15] H. M. Wark. A class of primary Banach spaces. J. Math. Anal. Appl., 326(2):1427-1436, 2007.

Richard Lechner, Institute of Analysis, Johannes Kepler University Linz, Altenberger Strasse 69, A-4040 Linz, Austria

E-mail address: richard.lechner@jku.at 